\title{
Economic evaluation of HIV treatments: The I.CO.N.A. cohort study
}

\author{
Monica Merito $^{\mathrm{a}, *}$, Andrea Bonaccorsi ${ }^{\mathrm{a}}$, Fabio Pammolli ${ }^{\mathrm{b}}$, Massimo Riccaboni ${ }^{\mathrm{b}}$, \\ Gianluca Baio ${ }^{\mathrm{b}}$, Claudio Arici ${ }^{\mathrm{c}}$, Antonella D’Arminio Monforte ${ }^{\mathrm{d}}$, Patrizio Pezzotti ${ }^{\mathrm{e}}$, \\ Dario Corsini ${ }^{\mathrm{f}}$, Andrea Tramarin ${ }^{\mathrm{g}}$, Roberto Cauda ${ }^{\mathrm{h}}$, \\ Vincenzo Colangeli ${ }^{\mathrm{i}}$, Giuseppe Pastore ${ }^{\mathrm{j}}$ \\ for the I.CO.N.A. Study Group \\ a Laboratory of Economics and Management, Sant'Anna School of Advanced Studies, \\ Piazza Martiri della Libertà 33, 56127 Pisa, Italia \\ ${ }^{\mathrm{b}}$ Faculty of Economics, University of Florence, Florence, Italy \\ ${ }^{\mathrm{c}}$ Infectious Diseases Department, Ospedali Riuniti di Bergamo, Bergamo, Italy \\ ${ }^{\mathrm{d}}$ Institute of Infectious and Tropical Diseases, University of Milan, L. Sacco Hospital, Milan, Italy \\ e Istituto Superiore di Sanità, Rome, Italy \\ ${ }^{\mathrm{f}}$ Informa srl, Rome, Italy \\ g Infectious Diseases Department, San Bortolo Hospital, Vicenza, Italy \\ h Infectious Diseases Clinic, Catholic University, Rome, Italy \\ i Institute of Infectious Diseases, University of Bologna, Bologna, Italy \\ ${ }^{\mathrm{j}}$ Institute of Infectious Diseases, University of Bari, Bari, Italy
}

\begin{abstract}
Objective: To describe the changes in costs of care for HIV-positive patients in Italy after the spread of antiretroviral combination therapies (HAART).

Methods: Five thousand four hundred and twenty-two patients from the I.CO.N.A. (Italian Cohort Naive Antiretrovirals) study were followed between 1997 and 2002. Costs included antiretroviral therapies (ART), hospital admissions, prophylaxis, and main laboratory examinations. The perspective was that of the National Health Service.

Results: Admission costs per person-year decreased from 2148 euro in 1997 to 256 in 2002, while the average annual costs of ART increased from 2145 to 3149 euro (1997 prices). From 1997 to1999, ART costs increased from 42.3 to $85.9 \%$ of the total, while admission costs decreased from 42.3 to $7.0 \%$ and prophylaxis from 7.3 to $1.7 \%$. The breakdown of ART costs shows how dual therapies decreased over time in favor of HAART, falling from $26.8 \%$ in 1997 to $5.9 \%$ in 2002. Patients with fewer than five treatment switches had the lowest costs distributions over the entire observation period.
\end{abstract}

\footnotetext{
* Corresponding author. Tel.: +39050 883281; fax: +39050883344.

E-mail address: merito@sssup.it (M. Merito).
} 
Conclusions: From 1997 to 2002 inpatient costs progressively decreased in favor of antiretroviral therapy. Annual average costs per patient decreased, while total direct costs increased over time: health resources, initially concentrated on hospitalized patients were then distributed over a growing number of subjects.

(C) 2005 Elsevier Ireland Ltd. All rights reserved.

Keywords: HIV infection; HAART; Health resources; Costs per person-year

\section{Introduction}

Since 1996, the use of powerful drug combinations to treat HIV/AIDS has become widespread in Western countries. Combinations of at least three different antiretroviral molecules, belonging to the class of nucleoside reverse transcriptase inhibitors (NRTI), protease inhibitors (PI), or non-nucleoside reverse transcriptase inhibitors (NNRTI), called HAART (Highly Active Antiretroviral Therapy), have radically changed the natural course of the HIV infection, as has been widely recognized in the clinical literature. Their theoretical efficacy in reducing the incidence of new AIDS cases and associated mortality, shown in controlled clinical studies [1,2], has since been confirmed in the population [3-6]. As a consequence, there has been a change in the use of health resources for HIV positive patients from inpatient to outpatient care, and from the use of drugs to treat opportunistic infections to antiviral medications [7-9].

The elevated cost of HAART, however, makes it important to investigate the impact of these combined therapies also in terms of the composition and total health expenses for the treatment of HIV/AIDS. Unfortunately, it is rather complex to determine with the necessary precision the financial burden and the clinical advantages associated with a single molecule of the combination administered. In addition, various health conditions and stages of the infection in patients at the start of treatment make the prescribed course of treatment notably different, therefore, it is preferable in an analysis such as this to concentrate on evaluating the costs of the drug combinations prescribed, and on the entire course of therapy for the individual. Taking these considerations into account, the objective of the present work is to describe the trend of direct health costs of HIV care in Italy after the introduction of HAART by examining the evolution of these costs in the I.CO.N.A. cohort study (Italian Cohort Naive Antiretrovirals).

\section{Methods}

\subsection{General characteristics of the I.CO.N.A. study}

The present study considered 5422 patients from the I.CO.N.A. cohort, enrolled between February 1997 and December 2002 in 69 Infectious Diseases Clinics. I.CO.N.A. is an observational study that includes adult patients with HIV infection naive to antiretrovirals (ARVs). Clinical and epidemiological details of the study have already been reported elsewhere [10]. The following is a partial list of data collected at enrollment: sex, age, risk factors for HIV infection, reasons for being antiretroviral naive, employment status, date and diagnosis of HIV-related and non HIV-related illnesses, Centers for Disease Control classification, CD4+ lymphocytes count and plasma HIV-RNA copies. The following data are then collected during the study: start and end dates of consecutive antiretroviral drug regimens and reasons for discontinuation; start and end date of prophylaxis and therapies for opportunistic infections; hospital admissions and death.

\subsection{Evaluation of costs}

Prospective routine data collection does not include information on health costs. The present work evaluated retrospectively costs incurred by the Italian National Health Service (SSN) for hospital admissions, drugs (antiretroviral, or for prophylaxis and treatment of opportunistic infections) and major laboratory examinations; all costs are expressed in euro.

The costs of antiretroviral therapies were calculated based on prices from the Italian National Pharmaceutical Formulary, adjusting for the average 
discount given at hospitals in the period of observation (varying between 27 and 55\%, according to the commercial product and the year considered). The average daily cost of each drug was calculated based on the daily dose usually administered, given standard dosage and number of tablets/capsules in each package. The average daily cost of the various drug combinations, calculated as the sum of each individual drug cost, was adjusted for variations in standard dosages in the presence of particular drug combinations, and then weighted for the total number of days of treatment in each year. The duration of each therapy was rounded up to the closest whole number of months, considering that the cost for the entire package of medications (usually corresponding to a month of treatment) is covered by SSN, even when therapy is interrupted early. Finally, annual cost of therapy per patient-year was reported. The costs of antiretroviral therapy are expressed both in current prices and in 1997 prices when compared to the other expenses categories. Prices were deflated using the general Consumer Price Index.

The costs of inpatient admissions were recovered applying national reimbursement rates [11], after linking the appropriate Diagnosis Related Group (DRG) to each admission on the basis of the discharge report, and considering inpatient length of stay. Annual cost of admissions was reported per person-year based on the entire cohort population.

Likewise, for immunological and virological examinations (lymphocytes subsets and viral load assessment) we referred to the rates established by the national formulary for ambulatory assistance services [12].

The costs of prophylaxis were based on retail prices from the Italian National Pharmaceutical Formulary, and reported in 1997 prices. When a chemical entity used in prophylaxis corresponded to more than one commercial product (whether branded or generic drugs) we used the price of the one with the highest market share. The daily average cost of each drug was calculated based on the daily dose most commonly prescribed, given the standard dosage and the number of tablets/capsules per package. The average daily cost was then weighted for the total number of treatment days for each year. In many cases the date of treatment completion was missing for prophylaxis and treatments for opportunistic infections; we substituted with the average duration of the same type of therapies reported in the study. Finally, the annual cost of prophylaxis per person-year was reported based on the entire cohort population.

\subsection{Statistical analysis}

The data refer to the period from February 1997 to December 2002. The trend of AIDS defining events (ADEs) and deaths was measured using the annual incidence rate (the number of cases for total person-years of the population followed in the year in question). The cumulative probability to discontinue the first, second, and following antiretroviral drug combinations was calculated using the Kaplan-Meyer method. Durations of antiretroviral therapies by number of treatment switches were compared using the log-rank test, which allows the statistical significance of differences to be evaluated. Distributions of antiretroviral therapies durations were compared in a box-plot of the length of treatments by number of switches.

\section{Results}

\subsection{Clinical outcomes}

The trend of mortality and ADEs per person-year in the 5422 subjects enrolled between 1997 and 2002 in the I.CO.N.A. cohort confirms the efficacy of HAART in reducing morbidity and mortality from HIV infection (Fig. 1). From 1997 to 2002 the number of ADEs per person-year in the I.CO.N.A. cohort decreased from 106 to 26 per thousand, and the number of deaths per person year from 29 to 8 per thousand. The incidence of hospital admissions in the cohort also decreased dramatically from 26.4 per thousand person-years in 1997 to 7.8 per thousand in 1998 , revealing values even lower than five per thousand from 1999 on. Similarly, length of stay drastically decreased from an annual average of 9.6 days per patient in 1997 to 0.9 days in 2002 .

\subsection{Antiretroviral therapies}

There were 3641 (67.2\%) patients in the cohort who followed at least one antiretroviral regimen between 1997 and 2002. No significant difference was found between the estimated duration of the first and second therapy, but the difference was significant between 


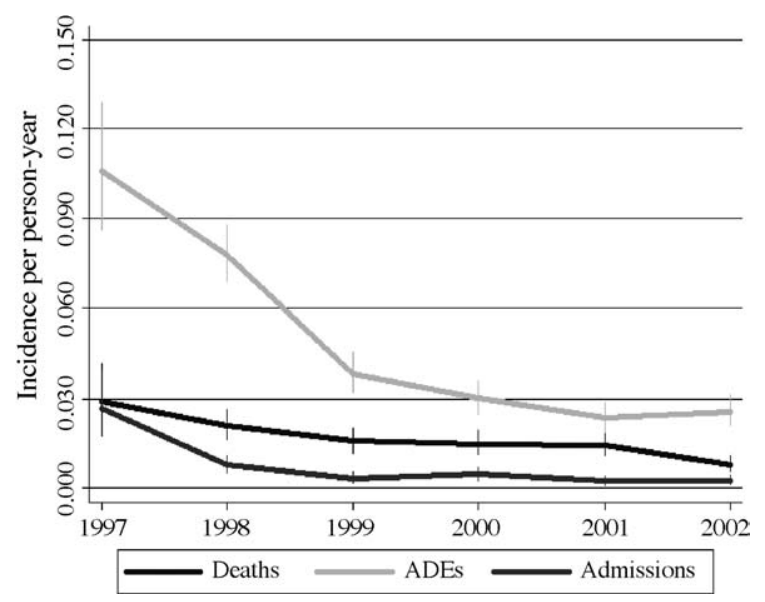

Fig. 1. Number of deaths, Aids defining events, and hospital admissions per person-year (95\% CIs).

the first two therapies compared with salvage therapies (median: 14 versus 11 months, $p<0.0001$ ). A box-plot of the duration, in months, of antiretroviral therapies by treatment switch is reported in Fig. 2 .

The patients who followed few therapies (maximum four switches in 6 years) show the lowest costs distributions over the entire observation period (standardized for the greater level of persistence of initial drug combinations); more atypical combinations, with much higher average annual costs, were seen in patients who received more therapies (Table 1).

Based on the frequency of each drug combination, characterized by number and class of molecules in

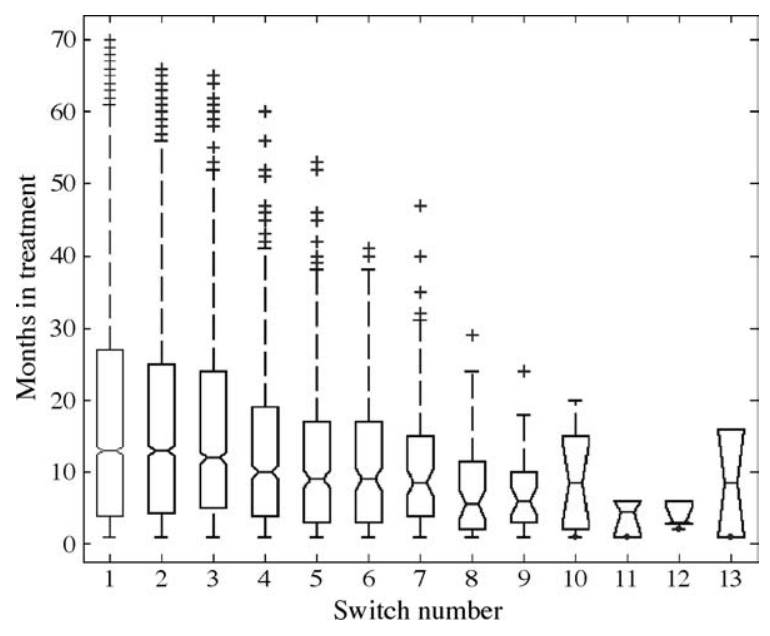

Fig. 2. Box-plot of therapy duration by treatment switch.

the mix, 13 common pharmacological combinations and one residual were identified. Table 2 reports the frequency (in percentages) of these drug mixes in total therapies started each year and Fig. 3 shows percentage costs of prevalent combinations over the 6 year period. Data reveals that the weight of dual therapies, still very common at the beginning of the study period, decreased over time both in terms of frequencies and costs in favor of HAART. From 2000, more diversified triple therapies were observed (corresponding to the introduction of new anti-HIV molecules to the market). In recent years, an increase in the incidence of so-called mega-HAART and atypical

Table 1

Average costs (euro) of antiretroviral therapy by number of treatment switches

\begin{tabular}{llllllll}
\hline $\begin{array}{l}\text { Number of } \\
\text { treatment switches }\end{array}$ & $\begin{array}{l}\text { Number of } \\
\text { patients }\end{array}$ & $\begin{array}{l}\text { Average } \\
\text { cost } 1997\end{array}$ & $\begin{array}{l}\text { Average } \\
\text { cost 1998 }\end{array}$ & $\begin{array}{l}\text { Average } \\
\text { cost 1999 }\end{array}$ & $\begin{array}{l}\text { Average } \\
\text { cost 2000 }\end{array}$ & $\begin{array}{l}\text { Average } \\
\text { cost 2001 }\end{array}$ & $\begin{array}{l}\text { Average } \\
\text { cost 2002 }\end{array}$ \\
\hline 1 & 1498 & 4914 & 5324 & 5528 & 5742 & 5923 & 6246 \\
2 & 882 & 5194 & 5579 & 5879 & 6066 & 6218 & 6237 \\
3 & 594 & 5201 & 5776 & 6201 & 6272 & 6331 & 6350 \\
4 & 325 & 5274 & 6004 & 6404 & 6551 & 6610 & 6724 \\
5 & 162 & 5568 & 6183 & 6694 & 6732 & 6796 & 6970 \\
6 & 89 & 5214 & 6143 & 6629 & 6680 & 6978 & 7327 \\
7 & 38 & 6296 & 6025 & 7072 & 7264 & 7216 & 7009 \\
8 & 22 & 5629 & 6394 & 6530 & 6668 & 6970 & 8153 \\
9 & 16 & 5015 & 6110 & 7079 & 7356 & 7076 & 7112 \\
10 & 8 & 4832 & 6759 & 6355 & 6876 & 6986 & 6745 \\
11 & 1 & 2996 & 5840 & 8757 & 7810 & 9666 & 8806 \\
12 & 3 & 2996 & 6939 & 6546 & 5655 & 6358 & 8278 \\
\hline
\end{tabular}


Table 2

Frequencies (percentages) of main antiretroviral regimens

\begin{tabular}{lrrrrrr}
\hline $\begin{array}{l}\text { Antiretroviral regimens } \\
\text { that include }\end{array}$ & 1997 & 1998 & 1999 & 2000 & 2001 & 2002 \\
\hline 1NRTI $^{\mathrm{a}}$ & 1.6 & 1.6 & 0.8 & 0.8 & 0.4 & 0.5 \\
1NRTI + 1NNRTI $^{\mathrm{b}}$ & 0.0 & 0.1 & 0.7 & 0.5 & 0.4 & 0.8 \\
1NRTI + 1NNRTI + 1PI $^{\mathrm{c}}$ & 0.0 & 0.8 & 2.4 & 0.9 & 0.4 & 0.3 \\
1NRTI + 1PI $^{\text {1NRTI + 2PI }}$ & 1.8 & 1.9 & 1.6 & 0.2 & 0.3 & 0.1 \\
2NRTI & 0.1 & 0.7 & 0.9 & 0.5 & 0.8 & 0.8 \\
2NRTI+2PI & 39.6 & 21.2 & 13.0 & 7.8 & 7.8 & 6.1 \\
2NRTI + NNRTI & 0.3 & 2.6 & 2.4 & 7.1 & 14.4 & 21.3 \\
2NRTI + NNRTI + PI & 0.1 & 8.5 & 27.3 & 45.4 & 41.0 & 31.9 \\
2NRTI + PI & 0.0 & 0.3 & 1.2 & 0.8 & 1.1 & 0.5 \\
3NRTI & 55.7 & 61.0 & 46.3 & 27.4 & 14.8 & 14.6 \\
3NRTI + NNRTI & 0.3 & 0.2 & 1.0 & 4.3 & 15.0 & 17.0 \\
3NRTI + PI & 0.0 & 0.0 & 0.1 & 0.7 & 0.6 & 1.6 \\
Other & 0.3 & 0.0 & 0.2 & 0.2 & 0.4 & 0.1 \\
Patients without ART (\%) & 0.3 & 1.0 & 2.0 & 3.2 & 2.8 & 4.3 \\
\end{tabular}

a NRTI: nucleoside reverse transcriptase inhibitor.

b NNRTI: nonnucleoside reverse transcriptase inhibitor.

c PI: protease inhibitor.

drug combinations has been reported. Data relative to 2 NRTIs and 2 PIs combinations instead reflect the spread of therapies with 2 PIs used in association.

Average costs per patient-year of prevalent antiretroviral therapies remained relatively stable over time, due to scarce price variations of the commercial products. In 2002 annual average costs per patient (that is, costs per patient-year taking ARVs) were 3520 euro for dual therapy, 6051 for HAART with one NNRTI,

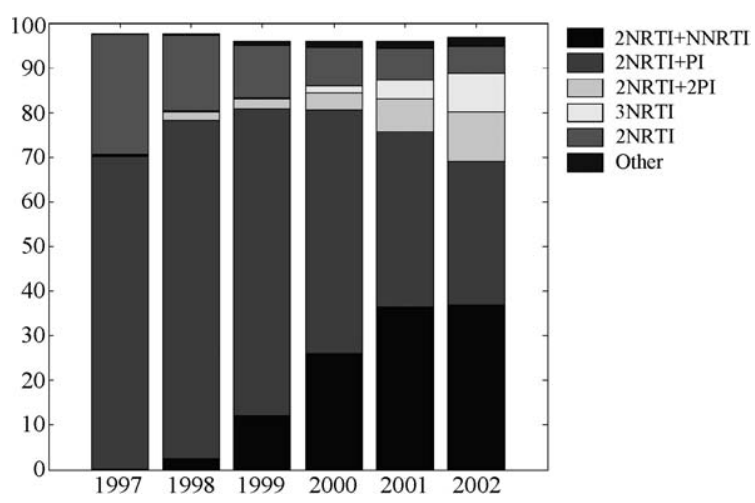

Fig. 3. Percentage incidence on total costs of prevalent antiretroviral therapies. NRTI: nucleoside reverse transcriptase inhibitor, NNRTI: nonnucleoside reverse transcriptase inhibitor, PI: protease inhibitor. "Other" refers exclusively to the drug combination defined as residual.
6474 for therapies with three NRTIs, 7299 for HAART with one PI, and 7634 for therapies with two PIs. The above costs include the $5 \%$ reduction in drug prices set forth by the government in April 2002. The data show however that total costs of ART per person-year (treated or not with antiretroviral medications) increased from 2145 euro in 1997 to 3149 euro in 2002 (1997 prices), testifying the passage from early therapies to increasingly more powerful and expensive combinations. The percentage of ART patients on total person-years in the cohort ranged from $40.9 \%$ in 1997 to $66.7 \%$ in 2000 .

Average cost difference among first, second and successive therapies decreased over time from 403 euro in 1998 (in 1997 the number of therapies from the third switch on was still too small to obtain a stable average) to 134 euro in 2001 (differences between costs increase again to 323 euro in 2002). Even if higher on average, costs of salvage therapies decreased over time, reaching levels of the first two therapeutic lines (from an average of 7315 euro in 1998 to 6903 euro in 2002). The average cost of second HAART decreased from 6974 euro in 1997 to 6609 in 2002, and since 2001 has been even lower than the average cost of first HAART, following the tendency to simplify antiretroviral combinations towards maintenance therapy in patients for whom the first therapy was effective.

The average annual costs of the remaining therapies (non HAART) increased slightly from 3215 euro per patient-year in 1997 to 3537 euro in 2002.

Costs per person-year not under ART increased from 2431 euro in1997 to 2793 in 2000 and then decreased again to 1904 euro in 2002. This last figure may be lower than average also because patients who were not reported as dropped before December 31, 2002 were considered still in follow-up until the end of the observation period, and drop-outs are recorded with some delay among patients without ART.

\subsection{Hospital admissions}

About $20 \%$ of patients had at least one hospital admission (15\% had a single admission, and 3\% two). In the univariate analysis, factors positively correlated to at least one admission were disease stage at enrollment and number of HIV and non-HIV related illnesses. Inpatient costs per person-year drastically fell after the spread of HAART in 1998, and continued 
to drop almost monotonically in the following years, decreasing from 2148 euro in 1997 to 256 euro in 2002.

In subjects treated with HAART, average inpatient costs decreased from 345 euro per year in 1997 to 205 in 2002. It is evident how the increasingly widespread use of HAART was the fundamental cause in reducing inpatient costs in the whole cohort.

\subsection{Therapies for opportunistic infections}

The costs of prophylaxis and treatments for opportunistic infections per person-year decreased from 371 euro in 1997 to 26 euro in $2002(-93 \%)$; the greatest reduction was found between 1997 and $1999(-80 \%)$. The composition of costs for opportunistic infections treatment remained similar over time, with a prevalence of herpetic viruses (on average $37 \%$ of the total), followed by mycosis (31\%) and mycobacteria (27\%); the costs for PCP (Pneumocisti Carinii Pneumonia) and toxoplasmosis were residuals.

\subsection{Laboratory examinations}

Laboratory expenses decreased almost constantly over the 6 years of observation from 409 euro in 1997, to 218 euro in 2002. In particular, costs for lymphocytes subsets decreased monotonically over time, falling from 81 to 42 euro per year between 1997 and 2002. In all, the costs for HIV-RNA decreased from 328 euro in 1997 to 176 in 2002 (showing a strong downturn at the beginning: $-33 \%$ between 1997 and 1998). In particular, the costs for laboratory examinations followed the same trend between first, second, and successive antiretroviral therapies; patients with three or more switches incurred the highest expenses (the difference in laboratory costs between patients in first line therapy and patients with at least three switches was 26 euro in 2002). The downward trend of laboratory expenses is due to the steady decrease in the average number of tests performed. In 1997, lymphocytes subsets and HIV-RNA assessments per person-year were 4.7 and 4.2, respectively, whereas by 2002 they had diminished to 2.4 and 2.3 , respectively.

\subsection{Total costs}

Total costs per person-year in the cohort decreased from 5073 euro in 1997 to 4226 in 1998; following which they increased, to 4581 euro in 2000, and decreased again to 3649 in 2002 . The incidence of hospital admissions, antiretroviral therapies, prophylaxis and treatments of opportunistic infections, and immunological and virological examinations on total costs changed markedly over time. In less than 2 years (from 1997 to 1999), costs for ART increased from 42.3 to $85.9 \%$ of the total, while costs for admissions decreased from 42.3 to $7.0 \%$; costs of prophylaxis and laboratory examinations changed from 7.3 and $8.1 \%$ in 1997 to $0.7 \%$ and $6.0 \%$ in 2002 , respectively.

\section{Discussion}

The I.CO.N.A. study collects prospective data coming from over 70 clinical centers in Italy that follow about $65 \%$ of the known HIV-positive population [13]. As already detailed in previous analyses, carried out in this [14] and other Italian studies [6,15-17], the diffusion from 1996 of antiretroviral combination therapies with three or more drugs (HAART) led to a marked decrease in mortality and incidence of AIDS cases.

This trend certainly led to a notable change in health costs. There are not many Italian studies on health resources consumption and costs of HIV-positive patients after the diffusion of HAART. A study conducted on 181 patients with less than $200 \mathrm{CD} 4 / \mathrm{mm}^{3}$ estimated that the average length of inpatient stays decreased from 18.9 days per person-year in 1996 to 15.6 in 1997 , following a 70\% reduction in new ADEs and a steady decrease in mortality. Total expenses remained similar in the 2 years considered, but moved from inpatient costs, to antiretroviral medications expenses [18]. A later multicenter observational study, with 483 patients followed from 1997 to 1998, reported a shorter length of stay (8.7 days per person-year) compared to previously published results, but similar to the estimates obtained in the present study, and ascribed this finding to a wider diffusion of HAART. The same study recorded ART costs per patient-year ranging from 1885 euro for asymptomatic subjects with more than $500 \mathrm{CD} 4 / \mathrm{mm}^{3}$ to 5353 euro for symptomatic patients [19]. In a recent study that retrospectively compared data of a cohort of symptomatic patients in 1994 with a similar cohort in 1998, a reduction of 3925 euro (1999 prices) in direct health costs per person-year was calculated. In 1998, ART costs per patient-year were equal to 7283 euro 
and accounted for $63.5 \%$ of total direct medical costs [20].

From the present study it emerges that, although expenses related to antiviral therapies increased by $34 \%$ per person-year, from 1997 to 2002 inpatient costs for HIV-positive patients noticeably decreased $(88 \%)$ in Italy. In particular, costs of hospital admissions per person-year were always inferior $(20 \%)$ in the population treated with HAART. HAART therapy permanently modified health resources consumption, moving it from the hospital to the patient's home: the observed reduction in inpatient expenses was the consequence of a lower incidence of ADEs and other opportunistic infections. In addition, the study shows that with the emergence of diagnostic standards for the new antiretroviral therapies, laboratory examinations costs decreased by $47 \%$ in 6 years. Paralleling the improvement in immuno-virologic parameters and clinical conditions of patients in the cohort is the almost complete disappearance, in terms of incidence on total costs, of prophylactic therapies and treatments for opportunistic diseases $(-93 \%)$.

It is difficult to compare such results with those obtained in other countries, due to the differences in the epidemiology of the HIV infection and of structural differences between health systems, in terms of type of health services offered and their accessibility, as well as health resources consumption implied. In a prospective, monocentric study on direct health costs in a German cohort of 201 HIV-positive patients enrolled in 1997, and with follow-up visits in 2000 and 2001, mean HAART costs per patient-year decreased significantly from 17,746 to 16,007 euro. Together with the reduction of expenditures for additional drugs, hospital admissions and diagnostic tests, this led to a 32\% decrease of total direct costs, from a mean of 35,865 euro per patient-year in 1997 to 24,482 euro in 2001 [21]. Another study, conducted on an observational cohort of Medicaid patients in Maryland (USA) followed between 1995 and 1997, similarly concluded that a PI containing regimen significantly reduced inpatient costs and expenses related to opportunistic infections, so that even if pharmaceutical expenses increased, overall health costs remained stable or slightly lower for these patients [8]. This tendency was confirmed by the HIV Cost and Services Utilization Study, for which 2864 patients were interviewed, representative of the entire HIV-positive adult population in
America that received assistance in 1996. As a result of the introduction of HAART, total cost per patient decreased by 10\% between 1996 and 1998 [9].

The costs of antiretroviral therapy computed in our work are in line with those reported by previous Italian studies, but notably lower than those found by other European and US researches. Since ARVs are covered by the SSN only if delivered at hospital, we valued antiretroviral drugs at hospital prices, which can be even less than half of retail prices (hospital prices were also used by other Italian studies $[19,20])$. In addition, the I.CO.N.A. cohort enrolls subjects who are still naive to ARVs. These patients are often in earlier stages of the disease and receive less expensive treatments compared to those included in many published works.

The introduction in Italy starting from 1995 of DRGs as the basis of a prospective reimbursement system for hospital services raised several issues at the beginning [22]. It was necessary to adopt a coding system for procedures and to produce a minimum discharge set for each acute care hospital stay. Major difficulties in the implementation of DRGs concerned lack of reliable information on production costs and of case-mix statistics. As a result, the compensation rates as to some DRGs provide an opportunity for profit, while the compensation rates for other DRGs do not cover the actual costs of providing those services. So far there has not been a specific debate on the adequacy of compensation rates for HIV care, whereas some concerns have arisen about the possible misclassification of cases. Some inpatient episodes regarding HIV positive subjects may be improperly assigned to Major Diagnostic Category (MDC) 25-HIV infection so as to benefit of the higher reimbursement rates for DRGs that fall under this diagnostic category.

The present study has some limitations. In the first place, the perspective was that of the SSN: thus, direct non-health costs (various out of pocket expenses, such as transportation) were not taken into consideration nor indirect costs associated to patients' productivity losses. Ignoring indirect costs may have underestimated the potential benefits of HAART. Secondly, the analysis did not include all health costs. It was impossible, for example, to distinguish visits to a specialist in an infectious diseases ward from day-hospital admissions; in addition, data on resistance tests were incomplete, and consultations with specialists from other wards were missing. Moreover, 
standard costs were used for the evaluation of hospital services (national DRGs rates). In theory, unit costs of hospital resources should instead be estimated based on a detailed examination of costs involving all hospitals participating in the study. The reimbursement rates might not accurately reflect real costs of HIV hospital care. Another limitation concerns the representativeness of the I.CO.N.A. cohort with respect to the adult HIV-positive population in Italy: in the first phase of enrollment above all, subjects in advanced phases of the infection, who, for whatever reason, had not yet been treated with antiretroviral drugs were over-represented in the sample. Finally, there is a problem in the cohort of temporary interruptions of follow-up: in these cases it is not usually possible to combine the separate treatment periods occurred at one or more participating hospitals. The date of administrative censoring was December 31, 2002, for all patients not lost to follow-up for any reason before that date (independently of the date of their last visit).

The present study is an analysis of the costs of HIV infection, which does not take into consideration the substantial clinical benefits of HAART, in terms of improvements in expected length and quality of life. We tried to retrospectively reconstruct the direct costs following a bottom-up approach, meaning estimating total health expenses from resources consumption data collected at the patient level; then, discounted costs, standardized for unit of time, and composition of total costs per expense category were calculated [23]. Full economic analyses [24] like those that compare costs and efficacy of antiretroviral therapies for specific patient groups will be the focus of future work. In particular, future studies may examine the impact in terms of costs and outcomes of important clinical choices, such as those relative to the time at which antiretroviral therapy should be started with respect to the level of immunosuppression reached or the initial drug combination. The analyses conducted to this point revealed that costs distributions for HIV treatments were substantially different among patients, based on number, type, and duration of therapies followed: more unstable expenses patterns seemed to be associated with patients who changed antiviral therapy often. Thus, in a cost analysis of HIV infection it would be important to identify and describe the clinical profile of patients linked to the most economically relevant therapeutic strategies, as well as research the principal determinants of the high costs of treatment and hospitalizations associated to specific patient profiles.

\section{Conclusions}

Despite the above limitations, this study provided important information on the costs of HIV patients management in Italy, due to the size of the observational sample considered, and the detailed information available, especially on antiretroviral therapies. Consistently with the recent international literature, the results of the analysis testified the change in the composition of expenses, characterized by the progressive reduction in inpatient costs in favor of antiretroviral therapy. Annual average costs per patient decreased: the reduction in the incidence of hospital admissions, combined with the administrative reduction in the prices of medications in 2002, offset the spread of more expensive antiretroviral therapies. Total direct costs instead seemed to increase over time, as a consequence of the fact that more patients were permanently treated and continued antiretroviral therapy for longer and longer periods of time. As a result of this trend, health resources first concentrated essentially only on hospitalized patients, were then distributed over a growing number of patients.

\section{Acknowledgement}

The I.CO.N.A. network is supported by an unrestricted educational grant provided by GlaxoSmithKline, Italy.

\section{Appendix A}

The members of the I.CO.N.A. Study Group are: Italy: Ancona: M Montroni, G Scalise, MC Braschi, MS Del Prete. Aviano (PN): U Tirelli, R Cinelli. Bari: G Pastore, N Ladisa, G Minafra. Bergamo: F Suter, C Arici. Bologna: F Chiodo, V Colangeli, C Fiorini, O Coronado. Brescia: G Carosi, GP Cadeo, C Torti, C Minardi, D Bertelli. Busto Arsizio: G Rizzardini, G Migliorino. Cagliari: PE Manconi, P Piano. Catanzaro: T Ferraro, A Scerbo. Chieti: E Pizzigallo, M D'Alessandro. Como: D Santoro, L Pusterla. Cremona: 
G Carnevale, D Galloni. Cuggiono: P Viganò, M Mena. Ferrara: F Ghinelli, L Sighinolfi. Firenze: F Leoncini, F Mazzotta, M Pozzi, S Lo Caputo. Foggia: G Angarano, B Grisorio, A Saracino, S Ferrara. Galatina (LE): P Grima, P Tundo. Genova: G Pagano, G Cassola, A Alessandrini, R Piscopo. Grosseto: M Toti, S Chigiotti. Latina: F Soscia, L Tacconi. Lecco: A Orani, P Perini. Lucca: A Scasso, A Vincenti. Macerata: F Chiodera, P Castelli. Mantova: A Scalzini, G Fibbia. Milano: M Moroni, A Lazzarin, A Cargnel, GM Vigevani, L Caggese, A d'Arminio Monforte, D Repetto, R Novati, A Galli, S Merli, C Pastecchia, MC Moioli. Modena: R Esposito, C Mussini. Napoli: N Abrescia, A Chirianni, CM Izzo, M Piazza, M De Marco, R Viglietti, E Manzillo, M Graf. Palermo: A Colomba, V Abbadessa, T Prestileo, S Mancuso. Parma: C Ferrari, P Pizzaferri. Pavia: G Filice, L Minoli, R Bruno, S Novati. Perugia: F Baldelli, M Tinca. Pesaro: E Petrelli, A Cioppi. Piacenza: F Alberici, A Ruggieri. Pisa: F Menichetti, C Martinelli. Potenza: C De Stefano, A La Gala. Ravenna: G Ballardini, E Briganti. Reggio Emilia: G Magnani, MA Ursitti. Rimini: M Arlotti, P Ortolani. Roma: R Cauda, F Dianzani, G Ippolito, A Antinori, G Antonucci, S D'Elia, P Narciso, N Petrosillo, V Vullo, A De Luca, S Di Giambenedetti, M Zaccarelli, R Acinapura, P De Longis, M Ciardi, G D’Offizi, MP Trotta, P Noto, M Lichtner, MR Capobianchi, E Girardi, P Pezzotti, G Rezza. Sassari: MS Mura, M Mannazzu. Taranto: F Resta, K Loso. Torino: P Caramello, A Sinicco, ML Soranzo, G Orofino, M Sciandra, M Bonasso. Varese: PA Grossi, C Basilico. Verbania: A Poggio, G Bottari. Venezia: E Raise, S Pasquinucci. Vicenza: F De Lalla, G Tositti. London, UK: A Cozzi Lepri.

\section{References}

[1] Hammer SM, Squires KE, Hughes MD, et al. A controlled trial of two nucleoside analogues plus Indinavir in persons with human immunodeficiency virus infection and CD4 cell counts of 200 per cubic millimeter or less. New England Journal of Medicine 1997;337(11):725-33.

[2] Montaner JSG, Reiss P, Cooper D, et al. A randomised, Double-blind Trial Comparing Combinations of Nevirapine, Didanosine, and Zidovudine for HIV-Infected Patients The INCAS Trial. Journal of the American Medical Association 1998;279(12):930-7.

[3] Palella Jr FJ, Delaney KM, Moorman AC, et al. Declining morbidity and mortality among patients with advanced human immunodeficiency virus infection. New England Journal of Medicine 1998;338(13):853-60.

[4] Mocroft A, Ledergerber B, Katlama C, et al. Decline in the AIDS and death rates in the EuroSIDA study: an observational study. Lancet 2003;362(9377):22-9.

[5] Porter K, Babiker A, Bhaskaran K, et al. Determinants of survival following HIV-1 seroconversion after the introduction of HAART. Lancet 2003;362(9392):1267-74.

[6] Pezzotti P, Napoli PA, Acciai S, et al. Increasing survival time after AIDS in Italy: the role of new combination antiretroviral therapies. Tuscany AIDS Study Group AIDS 1999; 13(2):249-55.

[7] Mouton Y, Alfandari S, Valette M, et al. Impact of protease inhibitors on AIDS-defining events and hospitalizations in 10 French AIDS reference centers. AIDS 1997;11:F101-5.

[8] Gebo KA, Chaisson RE, Folkemer JG, et al. Costs of HIV medical care in the era of highly active antiretroviral therapy. AIDS 1999;13:963-9.

[9] Bozzette SA, Joyce G, McCaffrey DF, et al. Expenditures for the care of HIV-infected patients in the era of highly active antiretroviral therapy. New England Journal of Medicine 2001;344(11):817-23.

[10] d'Arminio Monforte A, Arici C, Ippolito G, et al. Lo studio di coorte I.CO.N.A. (Italian Cohort Naive Antiretrovirals): caratteristiche all'arruolamento dei primi 1676 soggetti. Giornale Italiano dell'AIDS 1997;8(4):119-26.

[11] Decreto Ministeriale 30/06/1997. Aggiornamento delle tariffe delle prestazioni di assistenza ospedaliera, di cui al decreto ministeriale 14 dicembre 1994 e Tariffe delle prestazioni di riabilitazione ospedaliera erogate in regime di degenza ex D.M. 30.06.1997 espresse in euro. Gazzetta Ufficiale 08/09/1997.

[12] Decreto Ministeriale 22/07/1996. Prestazioni di assistenza specialistica ambulatoriale erogabili nell' ambito del SSN e relative tariffe. Gazzetta Ufficiale 14/09/1996.

[13] Pezzotti P, d'Arminio Manforte A, Bagarini R, et al. Antiretroviral therapy in HIV-infected individuals in clinical practice: are the criteria for initiating and choosing the type of drug regimen based only on immunologic and virologic values. European Journal of Epidemiology 2000;16:919-26.

[14] Cozzi Lepri A, Phillips AN, d'Arminio Manforte A, et al. When to start highly active antiretroviral therapy in chronically HIVinfected patients: evidence from the I.CO.N.A. study. AIDS 2001;15:983-90.

[15] Dorrucci M, Balducci M, Pezzetti P, et al. Temporal changes in the rate of progression to death among Italians with known date of HIV seroconversion: estimates of the population effect of treatment. Journal of Acquired Immune Deficiency Syndromes 1999;22(1):65-70.

[16] Porta D, Rapiti E, Forastiere F, et al. Changes in survival among people with AIDS in Lazio, Italy from 1993 to 1998, Lazio AIDS Surveillance Collaborative Group. AIDS 1999;13(15):2125-31.

[17] Pezzotti P, Dorrucci M, Donisi A, et al. Sopravvivenza, progressione in AIDS e livello di immunosoppressione in persone HIVpositive prima e dopo l'introduzione della terapia antiretrovirale di combinazione con tre o più farmaci (HAART). Epidemiologia e Prevenzione 2003;27:333-9. 
[18] Bruno R, Sacchi P, Patruno SFA, et al. HAART (Zidovudina, Lamivudina, Indinavir): analisi dei costi in una popolazione di pazienti HIV positivi con conta linfocitaria CD4+<200/mmc. Pharmacoeconomics-Italian Research Articles 1999;1(1):35-41.

[19] Garattini L, Tediosi F, Di Cintio E, et al. Resource utilization and hospital cost of HIV/AIDS care in Italy in the era of highly active antiretroviral therapy. AIDS Care 2001;13(6):733-41.

[20] Tramarin A, Campostrini S, Postma MJ, et al. A multicenter study of patient survival, disability, quality of life and cost of care. Among patients with AIDS in Northen Italy. Pharmacoeconomics 2004;22(1):43-53.
[21] Stoll M, Claes C, Schulte E, et al. Direct costs for the treatment of HIV-infection in a German cohort after the introduction of HAART. European Journal of Medical Research 2002;7:463-71.

[22] Taroni F. DRG/ROD e Nuovo Sistema di Finanziamento degli Ospedali. Roma: Il Pensiero Scientifico Editore 1996.

[23] Beck EJ, Miners AH, Tolley K. The cost of HIV treatment and care. A global review. Pharmacoeconomics 2001;19(1):1339.

[24] Sendi P, Palmer AJ, Gafni A, et al. Highly active antiretroviral therapy. Pharmacoeconomic issues in the management of HIV infection. Pharmacoeconomics 2001;19(7):709-13. 